\title{
ENFERMAGEM E EDUCAÇÃO PERMANENTE A DISTÂNCIA: O EXEMPLO DO PROJETO TELESSAÚDE BRASIL, NÚCLEO RIO DE JANEIRO*
}

Magda Guimarães de Araujo Faria ${ }^{1}$, Helena Maria Scherlowski Leal David ${ }^{2}$

\begin{abstract}
RESUMO: A educação permanente das equipes de saúde no Brasil é alvo de políticas de saúde. O Projeto Telessaúde Brasil é um exemplo de educação permanente a distância, voltada para profissionais da Atenção Básica de Saúde. O presente estudo tem por objetivo descrever a participação dos enfermeiros cadastrados no Projeto Telessaúde Brasil (núcleo RJ), na atividade de teleconferência. Trata-se de estudo quantitativo, com desenho longitudinal e observacional e caráter descritivo. Os dados foram obtidos por meio dos registros na base de dados do Projeto e sua delimitação temporal corresponde ao período de abril de 2009 a março de 2010. Foram realizadas 40 teleconferências de enfermagem a 669 participantes. A frequência mensal variou entre 8 participantes no mês de maio e 154 em novembro. Evidenciamos que, a partir de uma maior divulgação do Projeto, as participações aumentaram, o que demonstra que o profissional está receptivo a novas estratégias de aprendizado.
\end{abstract}

PALAVRAS-CHAVE: Educação a distância; Educação em enfermagem; Tele-enfermagem.

\section{NURSING AND PERMANENT DISTANCE EDUCATION: THE EXAMPLE OF THE TELESSAÚDE BRASIL PROJECT AT RIO DE JANEIRO}

\begin{abstract}
The continuing education of health teams in Brazil is the target of health policy. Telehealth Brazil Project is an example of distance continuing education, facing the Primary Health Care professionals. This study aims to describe the participation of nurses registered in the Telehealth Project Brazil (RJ nucleus), on a teleconference activity. It is a quantitative, observational and descriptive study with a longitudinal design. Data were obtained through records in the project database and its temporal delimitation corresponds to the period from April 2009 to March 2010. 40 teleconferences were held to 669 nursing participants. The frequency ranges between 8 participants in May and 154 in November. We demonstrated that, from a wider divulgation of the project, the attendance have increased, which shows that professionals are open to new learning strategies.
\end{abstract}

KEYWORDS: Distance Education; Nursing education; Tele-nursing.

\section{ENFERMERÍA Y EDUCACIÓN PERMANENTE A DISTANCIA: EL EJEMPLO DEL PROYECTO TELESSALUD BRASIL, NUCLEO RIO DE JANEIRO}

RESUMEN: La educación permanente de los equipos de salud en Brasil es alvo de políticas de salud. El Proyecto Telessalud Brasil es un ejemplo de educación permanente a distancia, direccionada a profesionales de la Atención Básica de Salud. El presente estudio tiene el objetivo de describir la participación de los enfermeros del catastro en el Proyecto Telessalud Brasil (núcleo RJ), en la actividad de teleconferencia. Se trata de estudio cuantitativo, con dibujo longitudinal, observacional y carácter descriptivo. Los datos fueron obtenidos por medio de los registros en la base de datos del Proyecto y su delimitación temporal corresponde al periodo de abril de 2009 a marzo de 2010. Fueron realizadas 40 teleconferencias de enfermería a 669 participantes. La frecuencia mensal ha variado entre 8 participantes en mes de mayo y 154 en noviembre. Se evidenció que, a partir de una mayor divulgación del Proyecto, las participaciones aumentaron, lo que muestra que el profesional está receptivo a nuevas estrategias de aprendizaje.

PALABRAS CLAVE: Educación a distancia; Educación en enfermería; Tele-enfermería.

\footnotetext{
*Artigo extraído de dissertação apresentada ao Programa de Pós-Graduação em Enfermagem da Universidade do Estado do Rio de Janeiro-UERJ em 2010.

${ }^{1}$ Enfermeira. Mestre em Enfermagem. Teleconsultor de Enfermagem do Projeto Telessaúde Brasil - Núcleo Rio de Janeiro.

${ }^{2}$ Enfermeira. Doutor em Saúde Pública. Professor Adjunto da UERJ. Coordenadora de Enfermagem do Projeto Telessaúde Brasil - Núcleo Rio de Janeiro.
}

Autor correspondente:

Magda Guimarães de Araujo Faria

Projeto Telessaúde Brasil, núcleo Rio de Janeiro

R. Boulevard 28 de setembro, 157 - 20551-030 - Rio de Janeiro- RJ-Brasil

Recebido: 20/05/10

E-mail: mag_araujo@yahoo.com.br

Aprovado: $15 / 11 / 10$ 


\section{INTRODUÇÃO}

Ao longo dos últimos anos, o modelo tradicional de ensino vem mostrando várias fragilidades. A partir da necessidade de reformulação deste método convencional de ensino, surgiu a proposta da educação a distância (EaD). Essa estratégia metodológica de ensino é bastante viável para atender as demandas inerentes da sociedade moderna ${ }^{(1)}$.

O conceito de EaD é amplo. De modo sucinto, podemos especificá-lo como o método pedagógico de ensino em que o educador e o educando não compartilham o mesmo ambiente físico ${ }^{(2)}$. Na área da saúde, a EaD está cada vez mais presente sob forma de cursos de aperfeiçoamento, extensão, educação permanente além dos cursos de graduação e especialização fornecidos por instituições de ensino a distância ${ }^{(3)}$.

A utilização da internet como meio de consultas e buscas, também se insere em meio à facilidade de busca de conhecimentos a distância. Por meio desse método, não há discriminação de grau de escolaridade. Assim, qualquer pessoa pode ter acesso a informações que, até décadas atrás, só seriam expostas em livros técnicos ${ }^{(4)}$.

$\mathrm{Na}$ área da saúde, a EaD é uma forma cada vez mais utilizada para inserir o profissional em um meio que o estimule a buscar novos conhecimentos, principalmente por meio de cursos de extensão, atualização e pós-graduação, além do compartilhamento de sua experiência prática ${ }^{(5)}$.

Em campos mais específicos, como a atuação do enfermeiro na Atenção Básica, estratégias de educação permanente se fazem indispensáveis para atualizar o profissional a respeito de seus conceitos e suas práticas. Além disso, os programas de educação permanente nos quais os profissionais de enfermagem se inserem possibilitam o desenvolvimento de habilidades específicas, tais como postura crítica, autoavaliação e autogestão ${ }^{(6)}$.

Em diversas partes do mundo já foi comprovada a eficácia da utilização da $\mathrm{EaD}$ como método de inserção de programas de educação permanente. Como exemplo deste fato, temos um estudo realizado com profissionais integrantes da Atenção Básica, lotados em áreas rurais do Reino Unido. O resultado comprovou que $69 \%$ dos entrevistados utilizaram ferramentas do programa de educação permanente a distância proposto naquele país ${ }^{(7)}$.

Visando a proposta pedagógica da inserção de novas tecnologias em ambiente de ensino, o Ministério da Saúde criou em 2006 o Programa Telessaúde
Brasil. No ano seguinte, o Estado do Rio de Janeiro foi escolhido para sediar um dos núcleos do projeto piloto do programa. As estratégias de ensino adotadas por esta iniciativa são baseadas em ferramentas que auxiliam o profissional da Atenção Básica na busca de conhecimentos ou atualizações sobre diversos temas em que está inserido. Entre elas está a atividade de teleconferência ${ }^{(8)}$.

A partir dessa perspectiva, o presente artigo tem como objetivo descrever a participação dos enfermeiros do Projeto Telessaúde Brasil, núcleo Rio de Janeiro, na atividade de teleconferência, identificando os meses de maior participação e traçando um perfil de uso do sistema pelos sujeitos.

\section{Educação a Distância e Educação Permanente em Enfermagem}

Na Enfermagem, a EaD ganha notoriedade nas faculdades. Algumas disciplinas da graduação de Enfermagem possuem a EaD como didática principal. Em um estudo realizado em uma faculdade particular do Rio de Janeiro, onde algumas disciplinas presentes no currículo de formação em Enfermagem foram ministradas on line, a experiência foi avaliada pelos próprios alunos e $45,9 \%$ do total afirmaram que o esclarecimento de dúvidas foi muito bom, mesmo utilizando o sistema de ensino não presencial ${ }^{(9)}$.

A educação permanente em saúde é um processo de aprendizagem cujo objetivo é a mudança das práticas de saúde, visando análise crítica e, consequentemente, inovações técnicas adequadas. Trata-se de uma abordagem que amplia a compreensão sobre a função social da educação para além do meramente instrumental ${ }^{(10)}$.

Nos últimos anos, a educação permanente tem sido objeto de inúmeros debates, principalmente quando focada no âmbito público. A busca por novos métodos educativos nas áreas médicas não pode priorizar apenas áreas específicas, mas o conhecimento como um todo. É exatamente nesse sentido que se configura a necessidade de interação entre diversos profissionais e diversos saberes, para que seja instituída a troca de conhecimento entre as equipes ${ }^{(11)}$.

No sentido de aperfeiçoar o conceito de educação permanente em saúde, é válido destacar a divergência de conceitos entre educação permanente e educação continuada. A primeira, já explorada nessa pesquisa, refere-se a uma população multidisciplinar, com metodologias dinâmicas, de fluxo contínuo de 
conhecimento, que visa aprimorar o profissional e modificar intervenções para ações mais compatíveis com a realidade de trabalho, objetivando sempre a qualidade de vida da população atendida ${ }^{(12)}$.

Em relação ao conceito de educação continuada, verificamos a presença da metodologia de transmissão do conhecimento. O público-alvo dessa modalidade busca aperfeiçoamento em áreas específicas. Além disso, essa modalidade estabelece, ainda, um tempo fixo para a obtenção do conhecimento desejado ${ }^{(12)}$.

Quando falamos do processo de educação permanente atrelada aos trabalhadores da Estratégia Saúde da Família (ESF), podemos utilizar três propostas metodológicas principais: a metodologia baseada em problemas, a autoinstrução e a EaD. Essa última não substitui as outras modalidades de educação permanente, ao contrário: ela soma conhecimento, estimula o profissional a buscar novas fontes de dados e se ajusta às necessidades da equipe, respeitando as limitações geográficas e temporais ${ }^{(13)}$.

O Projeto Telessaúde Brasil, Núcleo Rio de Janeiro (TSRJ) vem ao encontro às demandas de educação permanente, porém com um público-alvo específico de trabalhadores da Atenção Básica de Saúde, mais precisamente na ESF. O objetivo principal do projeto é estabelecer ações de auxílio aos profissionais da saúde e, sobretudo, promover um plano de educação permanente das equipes de saúde da família, visando a modificação nas práticas de trabalho, que resulte na melhora do atendimento ${ }^{(14)}$.

As ações propostas pelo Projeto Telessaúde estão ligadas intrinsecamente à utilização de tecnologias. A metodologia das atividades é baseada na $\mathrm{EaD}$ em saúde e, por isso, o uso da internet é fundamental para inserção do trabalhador. Por este motivo, o profissional inserido deverá possuir conhecimentos básicos de informática para acesso às ferramentas de serviço.

Dentre as ações realizadas pelo Projeto Telessaúde Brasil, destacam-se a implantação do serviço de informática, sendo cedidos ao município os kits compostos pelo computador e webcam; capacitação da equipe para manuseio dos recursos tecnológicos; formalização do recurso de teleconsultoria, que conta com profissionais qualificados que auxiliam a equipe de saúde nas dúvidas decorrentes do cotidiano profissional; e, por último, o serviço de teleconferência, na modalidade de webconferência, que funcionam como teleaulas interativas sobre conteúdo específico, com data previamente agendada e temas de interesse atual sugeridos pelas equipes de saúde.

\section{METODOLOGIA}

O estudo teve abordagem quantitativa, com desenho longitudinal e observacional e caráter descritivo. Objetivou documentar o processo de implantação e desenvolvimento das atividades de educação permanente a distância do Projeto TSRJ, focalizando a atividade de teleconferência dirigida aos profissionais da enfermagem, incluindo enfermeiros, técnicos e auxiliares de enfermagem atuantes na Atenção Básica de Saúde, mais precisamente na ESF do Estado do Rio de Janeiro. Apesar dessa especificidade, não é vedada a participação de outras categorias profissionais neste tipo de atividade.

O estudo foi aprovado pelo Comitê de Ética em Pesquisa do Hospital Universitário Pedro Ernesto (HUPE), sob o parecer n. 2551/2009. A utilização do termo de consentimento livre e esclarecido mostrou-se desnecessária, por tratar-se de pesquisa observacional de dados secundários, ou seja, foram analisados apenas os registros de participação.

Os dados foram obtidos no período entre abril de 2009 (data que marca o início da atividade de teleconferência realizada pelo Projeto TSRJ) e março de 2010, por meio do registro de frequência das teleconferências semanais. É válido ressaltar que a população do estudo abrange o quantitativo total de enfermeiros inscritos no projeto, sendo incluídos nesta análise apenas aqueles que participaram, em algum momento, de teleconferências de Enfermagem. Em meados de janeiro do ano de 2010, o núcleo RJ contava com 320 profissionais inscritos.

A estratégia adotada para a realização deste trabalho é baseada na realização de videoconferências semanais, com temáticas previamente aprovadas. A visualização do conteúdo pode ser realizada de forma síncrona, em tempo real de realização da atividade, ou, assíncrona, por meio do ambiente virtual do Projeto.

De modo síncrono, a discussão sobre os temas abordados nas videoconferências é feita a partir da ferramenta de chat ou, quando necessário, é utilizada a ferramenta de microfone pelos participantes, para maior interação com o teleconferencista. Na modalidade assíncrona não existe possibilidade de interação imediata; porém, o participante pode trocar informação com o teleconferencista, por correio eletrônico.

A base para estas teleconferências requer, 
por parte dos participantes, apenas o computador com acesso à internet de banda larga, um programa navegador, alto-falantes para a escuta das apresentações - sendo desejável também a instalação de uma vídeo-câmera comum e microfone, para fins de interação. Contudo, apenas o acesso à internet com escuta é suficiente para que os profissionais assistam à teleconferência, neste caso interagindo por meio de um chat em tempo real que permanece acessível durante toda atividade.

\section{RESULTADOS}

No período analisado, foram realizadas 40 teleconferências de enfermagem com temáticas previamente aprovadas e a frequência de participação em teleconferências atingiu 215 pontos de acesso e 669 trabalhadores conectados em tempo real e, 3913 expectadores no ambiente virtual de aprendizagem.

Os pontos descritos acima correspondem ao número de computadores que estavam ligados à teleconferência no momento em que a mesma ocorreu. Quando tratamos da média de participantes mensais na modalidade síncrona, obtemos o índice de 56 pessoas.

A distribuição das frequências na atividade de teleconferência durante o período estudado está evidenciada de acordo com a tabela 1 e gráfico 1 .

Tabela 1 - Distribuição da frequência de pontos, enfermeiros e acessos ao ambiente virtual na atividade de teleconferência de Enfermagem. Rio de Janeiro, 2010

\begin{tabular}{lccc}
\hline Meses & Pontos & $\begin{array}{c}\text { Participantes } \\
\text { síncronos }\end{array}$ & $\begin{array}{c}\text { Acessos no } \\
\text { ambiente } \\
\text { virtual }\end{array}$ \\
\hline Abril - 2009 & 3 & 16 & 43 \\
Maio - 2009 & 3 & 8 & 213 \\
Junho - 2009 & 33 & 86 & 639 \\
Julho - 2009 & 18 & 83 & 290 \\
Agosto - 2009 & 42 & 77 & 262 \\
Setembro - 2009 & 18 & 66 & 641 \\
Outubro - 2009 & 9 & 35 & 334 \\
Novembro - 2009 & 30 & 154 & 151 \\
Dezembro - 2009 & 13 & 32 & 115 \\
Janeiro - 2010 & 14 & 29 & 728 \\
Fevereiro - 2010 & 9 & 34 & 204 \\
Março - 2010 & 23 & 50 & 293 \\
Total & $\mathbf{2 1 5}$ & $\mathbf{6 6 9}$ & $\mathbf{3 9 1 3}$ \\
\hline
\end{tabular}

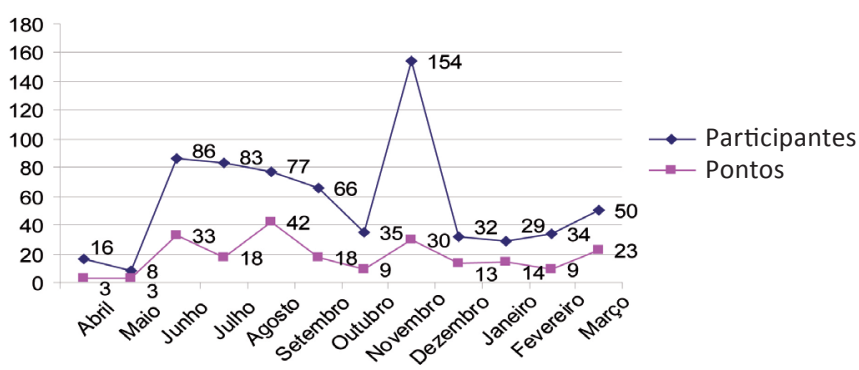

Gráfico 1 - Distribuição da frequência de enfermeiros na estratégia de teleconferência de Enfermagem. Rio de Janeiro, 2010

Em relação às perguntas realizadas durante o período de teleconferência, foram registradas 56 dúvidas, sendo distribuídas da seguinte forma: $9 \mathrm{em}$ junho, 2 em julho, 15 em agosto, 1 em setembro, 1 em outubro e 5 em novembro. No ano de 2010, tivemos 9 questionamentos em janeiro, 5 em fevereiro e $11 \mathrm{em}$ março.

\section{DISCUSSÃO}

O quantitativo de participantes mensais foi maior em nosso estudo quando comparado à média de enfermeiros participantes na experiência de teleconferências e webconferências apontada por pesquisa realizada a partir de um curso sobre enfermagem oncológica, que foi de 50 enfermeiros ${ }^{(15)}$.

É relevante frisarmos o alto índice de profissionais que acessam as teleconferências por meio do ambiente virtual de aprendizagem. Esse padrão nos permite traçar duas hipóteses: a primeira diz respeito à importância do conhecimento adquirido, o que leva o profissional a rever aulas que julgue essenciais para sua atividade. A segunda diz respeito à impossibilidade do enfermeiro em participar da atividade em tempo real, já que a atividade é realizada no horário de funcionamento das unidades de saúde.

O grande diferencial da participação dos profissionais na estratégia de teleconferência iniciou no mês de junho, pois, além da elevada presença, os profissionais começaram a participar ativamente dos momentos de discussão, expressando suas ideias, opiniões e expondo dúvidas, situação inédita até o momento.

No mês de agosto, notamos um leve declínio nas participações, sendo agravado em setembro e outubro. Exatamente no mês de outubro percebemos o maior declínio nas participações, o que pode ser atribuído ao número de feriados do referido mês; com 
isso, tivemos apenas duas teleconferências, culminando em uma baixa do número total de participantes.

No mês de novembro registramos o maior número de participantes em uma única teleconferência, 79 pessoas. A procura pode ser referida devido à familiaridade com a demanda encontrada pelos profissionais da atenção básica de saúde. O tema trabalhado foi "Adesão ao tratamento da hipertensão e diabetes", situação que representa uma parte importante do papel do enfermeiro da Atenção Básica ${ }^{(16)}$.

Em dezembro, percebemos um declínio em relação ao mês anterior. Tal fenômeno pode ser associado a esta época do ano, em que ocorrem festividades, e também porque vários profissionais saem de férias.

No ano seguinte, em 2010, logo em janeiro foi observada uma queda brusca no quantitativo de participantes, porém o acesso ao ambiente virtual de aprendizado elevou-se consideravelmente se comparado ao mês anterior. Atribuímos esta situação devido à época do ano, sendo comumente o mês preferido pelos profissionais para férias. A partir do mês de fevereiro, observamos um leve aumento do número de expectadores da atividade de teleconferência. No mês de março, as participações aumentaram ainda mais, porém ainda não atingindo a média mensal de participantes.

Quando falamos de participações no ano de 2010, percebemos que $26 \%$ das participações ocorreram no mês de janeiro, enquanto que em fevereiro esse percentual subiu para $30 \%$ e em março para $44 \%$. Acreditamos na tendência de aumento desse percentual com o passar dos meses do ano.

Quando analisamos a participação e sua relação com atividades presenciais de mobilização e apresentação do Projeto, realizadas pelo Núcleo em algumas regiões fluminenses durante 2009, verifica-se um aumento na participação após a realização destas atividades durante os meses de maio, junho, julho e setembro.

As atividades presenciais, também chamadas de workshops, são de extrema importância para o aumento efetivo da participação dos profissionais ao Projeto Telessaúde. Nesse tipo de atividade, profissionais de diversas áreas aprendem a utilizar as ferramentas do Projeto e realizam o cadastramento no sistema.

No mês de maio foi realizado pela equipe, o primeiro workshop na Região Médio Paraíba. Atribuímos este fato ao aumento considerável dos participantes nas teleconferências a partir do mês de junho. O mesmo ocorreu na Região Serrana em junho e na Região Metropolitana do Município do Rio de Janeiro em setembro.

A área que teve maior participação nas atividades propostas foi a Região Metropolitana, com 36,1\% de participantes; as regiões do Médio Paraíba e Centro-Sul Fluminense obtiveram índices semelhantes, com aproximadamente 16,5\%; o Noroeste Fluminense foi responsável por $10 \%$ das participações; as regiões da Baixada Litorânea e Serrana também tiveram índices semelhantes, $6,6 \%$ cada. A Região do Norte Fluminense teve o índice de 1,8\%, enquanto a Região da Baía de Ilha Grande obteve apenas 0,3\%. Além disso, devemos lembrar daqueles que, por algum motivo, não especificaram a área onde residem, a estes foram computadas $5,2 \%$ das participações.

A expressiva participação de profissionais da Região Metropolitana pode ser explicada pelo fato de ser a região com maior densidade numérica de profissionais e, possivelmente, por alguns outros fatores: maior interação e comunicação entre gestão e equipes; familiaridade com o uso da internet; por ter esta experiência se iniciado pelas capitais dos estados, e só posteriormente sendo ofertada para os municípios do interior; dificuldade maior de acesso em alguns municípios cujas equipes estão lotadas em áreas rurais e distantes. Todos estes fatores carecem de ser adequadamente investigados, constituindo-se, por enquanto, em meras hipóteses explicativas.

Em relação à acessibilidade por parte dos municípios, o Estado do Rio de Janeiro marca por seu numero reduzido de municípios, comparativamente a outros estados, e pela forte urbanização, o que garante condições mínimas adequadas para a instalação dos pontos de acesso do Telessaúde. No entanto, a partir de alguns relatos durante encontros presenciais com os enfermeiros, constatou-se que, no caso das equipes localizadas em áreas rurais, a dificuldade de acesso à internet pode se constituir em um impeditivo à participação pela necessidade de ter de se deslocar para um ponto de acesso mais central. Por outro lado, a democratização do acesso à internet por parte de alguns municípios, que investem na disponibilização de computadores públicos ou quiosques digitais, surge como uma perspectiva capaz de ampliar a oportunidade para acesso remoto.

Apesar do crescente número de participantes, a realidade da $\mathrm{EaD}$ relacionada à educação de enfermagem ainda se apresenta pouco expressiva em termos de demandas e de participação. Em uma pesquisa sobre um curso on line de cardioversão, o 
índice de alunos que terminaram o curso foi de $52,4 \%$ e a principal justificativa para evasão do curso foi a falta de tempo para dedicar-se às tarefas exigidas como método avaliativos ${ }^{(17)}$. Em outro estudo, dessa vez sobre esterilização de materiais, o índice de enfermeiros que finalizaram o curso foi de $78,6 \%{ }^{(18)}$.

Soma-se a esta situação o fato de que, na Atenção Básica, é ainda mais recente a utilização de recursos de $\mathrm{EaD}$ mediada pela internet por parte dos profissionais de saúde. A expansão do acesso à internet, como forma de inclusão digital, encontra ainda barreiras tecnológicas, em especial a restrição aos grandes centros urbanos da oferta de acesso à internet com velocidade que permita o uso adequado das mídias visuais. Aos municípios do interior dos estados restam alternativas pouco estáveis de acesso por rádio, sendo que ainda persiste, em muitos deles, o acesso discado, de baixa velocidade.

Em relação às perguntas realizadas durante o período de teleconferência, evidencia-se, que não é o número de participantes que determina maior participação, em termos de interação, durante a teleconferência, e que esta interação parece estar antes relacionada à temática e à mobilização que esta produz nos participantes.

\section{CONCLUSÃo}

A educação a distância é uma das principais maneiras de auxiliar a prática de saúde, pois propicia ao profissional o conhecimento sobre determinado assunto e instiga o mesmo a buscar maiores informações, utilizando os diversos recursos disponíveis por meio dessa modalidade.

$\mathrm{Na}$ ESF, os programas de educação permanente, quando aliados a modelos a distância, são de extrema valia, pois desse modo é possível atender a profissionais que até então tinham que ultrapassar barreiras geográficas e temporais para sua qualificação profissional.

A experiência na área de enfermagem do Projeto TSRJ demonstra claramente a necessidade dos profissionais da Atenção Básica por programas de educação permanente a distância.

Acreditamos que, com a devida divulgação, a tendência do Projeto é de que tenha maior adesão com o passar dos meses. A configuração do modelo de teleconferências semanais e a divulgação por correio eletrônico dos temas previamente definidos têm contribuído para a maior participação dos enfermeiros em nossas atividades.

\section{REFERÊNCIAS}

1. Belloni ML. Educação a distância. $5^{\mathrm{a}}$ ed. Campinas: Editora Autores Associados; 2008.

2. Brasil. Associação Brasileira de Educação a Distância - ABED. Sobre a EAD [acesso em 5 de mar 2010]. Disponível: http://tiny.cc/3li5i

3. Rodrigues RCV, Peres HHC. Panorama brasileiro do ensino de enfermagem on line. Rev Esc Enferm USP. [Internet] 2008; 42(2) [acesso em 5 mar 2010]. Disponível: http://tiny.cc/km7nt

4. Alves VLS, Cunha ICKO, Marin HF, Oliveira O. Criação de um website para enfermeiros sobre o pé diabético. Acta Paul Enferm [Internet] 2006; 19(1) [acesso em 5 mar 2010]. Disponível: http://www.scielo. br/pdf/ape/v19n1/a09v19n1.pdf

5. Ortiz MCL, Ribeiro RP, Garanhani ML. Educação a distância: uma ferramenta para educação permanente de enfermeiros que trabalham com assistência perioperatória. Cogitare Enferm. [Internet] 2008; 13(4) [acesso em 18 mai 2010]. Disponível: http://tiny. $\mathrm{cc} / \mathrm{khauu}$

6. Oliveira RG, Marcon SS. Trabalhar com famílias no programa de saúde da família: a prática do enfermeiro em Maringá-Paraná. Rev Esc Enferm USP. [Internet] 2007; 41(1) [acesso em 5 mar 2010]. Disponível: http:// tiny.cc $/ 5 \mathrm{fh} 7 \mathrm{z}$

7. Richards H, King G, Reid M, Selvaraj S, McNicol I, Brebner E et al. Remote working: survey of attitudes to eHealth of doctors and nurses in rural general practices in the United Kingdom. Fam Pract [Internet] 2005; 22(1) [acesso em 5 mar 2010]. Disponível: http://tiny.cc/n9qlu

8. Ministério da Saúde (BR). Programa Nacional de Telessaúde. Sobre o Programa [acesso em 5 mar 2010]. Disponível: http://tiny.cc/yzjyz

9. Camacho ACLF. Educação a distância na disciplina de legislação, ética e exercício de enfermagem. Rev Bras Enferm [Internet] 2009; 62(1) [acesso em 8 mar 2010]. Disponível: http://tiny.cc/1xq24

10. Ceccin RB. Educação permanente em saúde: descentralização e disseminação da capacidade pedagógica em saúde. Ciência \& Saúde Colet. [Internet] 2005; 10(4) [acesso em 8 mar 2010]. Disponível: http://tiny.cc/v28fp 
11. Batista RS, Batista RS. Os anéis da serpente: a aprendizagem baseada em problemas e as sociedades de controle. Rev Ciênc \& Saúde Colet. [Internet] 2009; 14(4) [acesso em 8 mar 2010]. Disponível: http://tiny. cc/urjc1

12. Mancia JR, Cabral LC, Koerich MS. Educação permanente no contexto da enfermagem e na saúde. Rev Bras Enferm [Internet] 2004;57(5) [acesso em 8 mar 2010]. Disponível: http://tiny.cc/2zak7

13. Ministério da Saúde (BR). Cadernos de atenção básica: Programa de Saúde da Família e a Educação permanente. Brasília: Ministério da Saúde; 2000.

14. Ministério da Saúde (BR). Portaria n. 35, de 4 de janeiro de 2007. Institui no âmbito do Ministério da Saúde, o Programa Nacional de Telessaúde. Diário Oficial da República Federativa do Brasil. Brasília, 05 jan 2007.

15. Gundim RS, Maranho IC. Relato de experiência: I Curso de enfermagem oncológica por teleconferência do Instituto de Ensino e Pesquisa; 2005 Jun. p. 20-4; Cidade do México, México; 2005.

16. Nascimento MS, Nascimento MAA. A prática da enfermeira no Programa de Saúde da Família: a interface da vigilância da saúde versus as ações programáticas em saúde. Rev Ciênc Saúde Colet. [Internet] 2005; 10(2) [acesso em 8 mar 2010]. Disponível: http://tiny.cc/e0nab

17. Sanches LMP, Lopes MHBM. Educação a distância sobre cardioversão e desfibrilação para enfermeiros. Rev Bras Enferm [Internet] 2008; 61(5) [acesso em 8 mar 2010]. Disponível: http://tiny.cc/79pwv

18. Quelhas MCF, Lopes MHBM, Ropoli EA. Educação a distância em processos de esterilização de materiais. Rev Esc Enferm USP [Internet] 2008; 42(4) [acesso em 8 mar 2010]. Disponível: http://tiny.cc/2zak7 